\title{
23-gauge vitrectomy assisted by combined endoscopy and a wide-angle viewing system for retinal detachment with severe penetrating corneal injury: a case report
}

\author{
This article was published in the following Dove Press journal: \\ Clinical Ophthalmology \\ 13 December 2011 \\ Number of times this article has been viewed
}

\section{Seita Morishita \\ Mihori Kita \\ Shin Yoshitake \\ Miou Hirose \\ Hideyasu Oh}

Department of Ophthalmology, Hyogo Prefectural Amagasaki

Hospital, Amagasaki, Hyogo, Japan
Correspondence: Mihori Kita

Department of Ophthalmology, Hyogo Prefectural Amagasaki Hospital,

I-I-I, Higashidaimotsu-cho, Amagasaki, Hyogo 660-0828, Japan

Tel +8166482 I52।

Fax +81664827430

Email mihorik@kuhp.kyoto-u.ac.jp
Background: We report a case of traumatic retinal detachment in an eye with severe corneal opacity that was successfully treated using 23 gauge $(G)$ transconjunctival vitrectomy assisted by endoscope and a wide-angle viewing system.

Case presentation: A 22-year-old Japanese man was referred to our hospital with the suspicion of traumatic retinal detachment of the right eye, 1 month after an open globe eye injury due to fireworks. At the time of his first visit, his best-corrected visual acuity was hand motion in the right eye. A $23 \mathrm{G}$ three port pars plana vitrectomy was conducted in combination with ophthalmic endoscope and a wide-angle viewing system. Endoscopy revealed a retinal detachment in the inferior quadrant with tiny retinal breaks. Primary reattachment of the retina was achieved by tamponade of $\mathrm{SF}_{6}$ gas. Five months after the vitrectomy, penetrating keratoplasty was performed and visual acuity recovered to 0.02 . Optical coherent tomography revealed thinning of the retina, which might be the cause of the remaining poor vision.

Conclusion: $23 \mathrm{G}$ vitrectomy assisted by combined endoscopy and a wide-angle viewing system could be advantageous in managing visualization constraints due to penetrating trauma.

Keywords: penetrating injury, vitrectomy, endoscope, wide angle viewing system, 23 gauge vitrectomy

\section{Introduction}

Recent technological advances in vitreous surgery systems, including microincision vitrectomy surgery and wide-angle viewing systems, have allowed safer and less invasive vitreoretinal surgeries. However, there is difficulty in performing vitrectomy due to visualization constraints. We encountered a case of traumatic retinal detachment with corneal opacity by injury due to fireworks, and the patient was successfully treated with a 23 gauge $(\mathrm{G})$ transconjunctival vitrectomy combined with endoscopy and a wide-angle viewing system.

\section{Case presentation}

A 22-year-old Japanese man in good general health was referred to our hospital after treatment at two other hospitals, with suspicion of traumatic retinal detachment of the right eye, 1 month after an open globe eye injury due to fireworks. Primary corneal and scleral sutures for multiple penetrating wounds had been performed as emergency procedures in the first hospital. No foreign bodies were discovered on preoperative computed tomography. Two weeks after the injury, a lensectomy was performed at the 
second hospital because of a shallow anterior chamber due to lens swelling.

At the time of his first visit, his best-corrected visual acuity was hand motion in the right eye and 1.2 in the left eye. The intraocular pressure in the right eye was low, although it was difficult to measure. Slit lamp examination revealed severe corneal opacity and edema, and a rough surface of the cornea (Figure 1) without iridocyclitis, hyphema, fibrin exudates, or any infectious signs in the anterior chamber. The corneal and scleral wounds were well adapted, and no fluid leakage was observed. The ocular fundus of the right eye was invisible due to corneal opacity and media opacity, but B-mode ultrasonography implicated a retinal detachment. The electroretinogram in the right eye was subnormal. The ocular findings in the left eye were normal and the patient had no symptoms of sympathetic ophthalmia.

A $23 \mathrm{G}$ three port pars plana vitrectomy was conducted in combination with ophthalmic endoscopy (Fiber Tec, Tokyo, Japan) and a wide-angle viewing system (Resight ${ }^{\circledR}$, Carl Zeiss, Oberkochen, Germany). First, viscoelastic materials were injected into the anterior chamber and anterior vitreous cavity through a limbal wound made with a $20 \mathrm{G}$ microvitreoretinal blade to create an opaque-free space for the endoscope. Endoscopy through the limbal wound and pupil revealed a large amount of swollen lens remnant, dehemoglobinized vitreous hemorrhage, and an intact posterior pole retina. Three $23 \mathrm{G}$ transconjunctival sclerotomy ports were created $3.5 \mathrm{~mm}$ posterior to the limbus under endoscopic observation. Removal of the lens remnant was performed with a vitreous cutter under endoscopic view, followed by removal of the dense vitreous opacity under the wide-angle viewing system through the clearer inferior-temporal portion of the cornea (Figure 2). Peripheral vitrectomy was conducted under endoscopic guidance without scleral indentation or

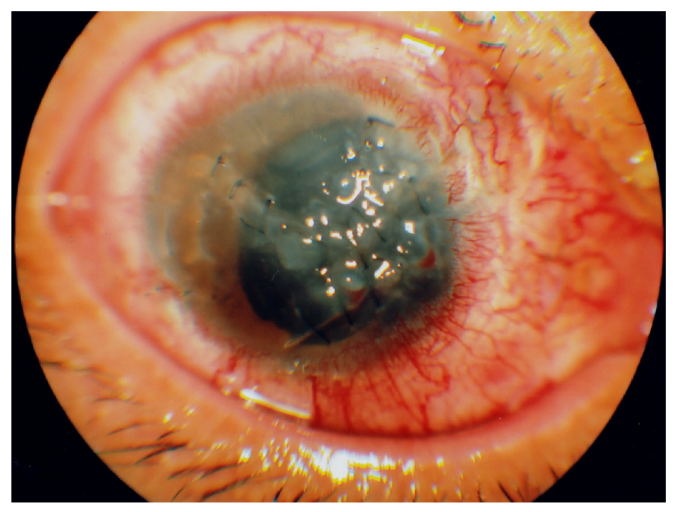

Figure I Anterior segment of the right eye at first visit. Note the rough surface and opacity of the cornea due to multiple penetrating injuries.

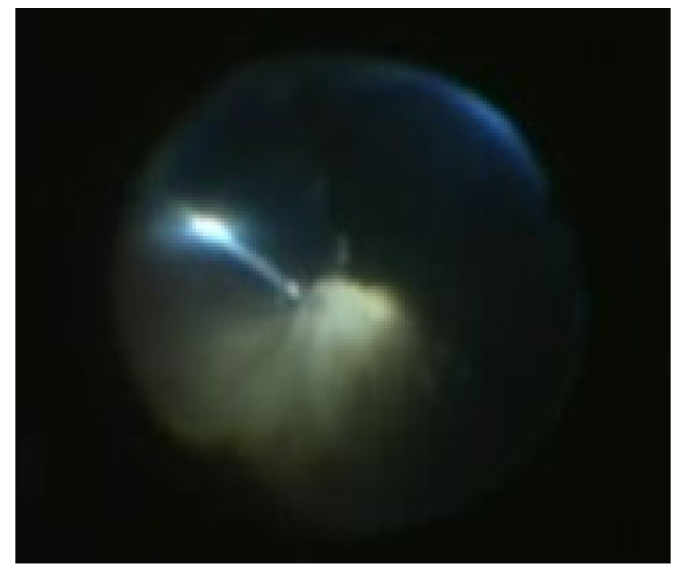

Figure 2 Dense vitreous opacity was removed with a wide-angle viewing system through the clearer inferior-temporal portion of the cornea.

anterior manipulations, and a retinal detachment in the inferior quadrant with tiny retinal breaks at the 5:00-6:00 portion of the periphery was identified. Moreover, vitreous incarcerations into the scleral perforation wounds were observed and excised under endoscopic view (Figure 3). Flattening of the retina was achieved by fluid-air exchange under a wide-angle viewing system, and internal drainage through the original break and retinopexy with external cryotherapy and supplementary endolaser was conducted under endoscopic view. No leakage of fluid from the penetrating injury wounds was observed during the procedures. Twenty percent $\mathrm{SF}_{6}$ gas was used to fill the vitreous cavity as an internal tamponade. The patient was encouraged to maintain a face-down position for 2 weeks. No intraoperative or postoperative complications were encountered.

The best-corrected visual acuity of the right eye recovered to 0.01 . Five months after vitrectomy, penetrating keratoplasty was performed (Figure 4) in another hospital, which allowed us to make ophthalmoscopic and optical

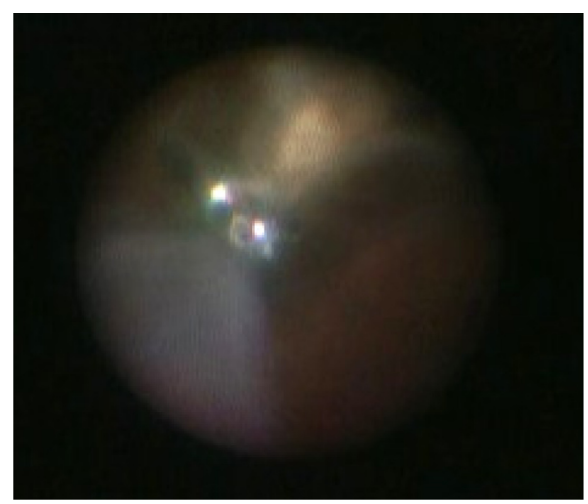

Figure 3 Vitreous incarcerations into the scleral perforation wounds were observed and were excised under the endoscopic view. 


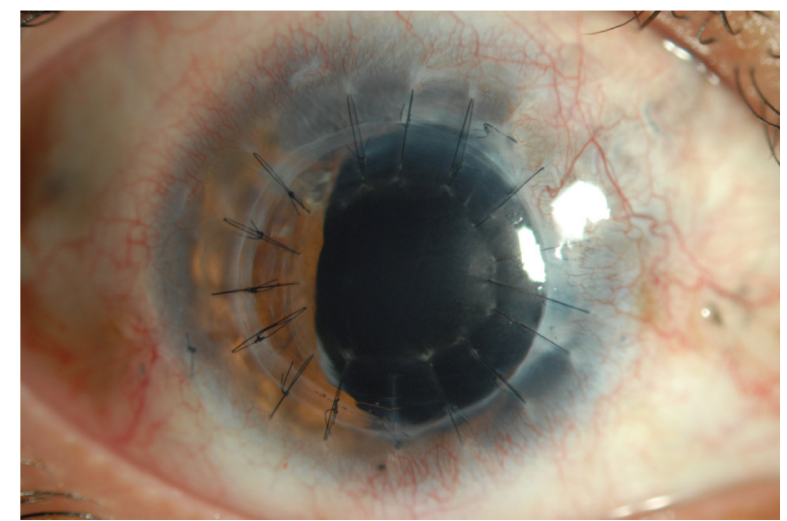

Figure 4 Photograph of anterior segment of the right eye 2 months after keratoplasty.

coherence tomography (OCT) observations, but the visual acuity in the right eye stayed at 0.02 . OCT revealed thinning of the retina posterior pole (Figure 5), which might be the cause of the remaining poor vision. Perimetry after keratoplasty showed relative central scotoma of the right eye in the area of thinning of the retina. At 10-month follow-up, the retina remained attached, the cornea graft remained clear, and the fellow eye had no symptoms of sympathetic ophthalmia.

\section{Discussion}

In severe penetrating corneal injuries, vitrectomy using a temporary keratoprosthesis during pars plana vitrectomy followed by keratoplasty has been thought to be beneficial. ${ }^{1-3}$ However, combined pars plana vitrectomy and simultaneous keratoplasty might cause intraoperative complications, including suprachoroidal hemorrhage and corneal graft failure. ${ }^{4}$ Recently, a two-step procedure comprising endoscope-guided vitrectomy followed by corneal transplantation has been reported. ${ }^{5,6}$ In our case, the cornea specialist at the second hospital pointed out that the risk of graft failure

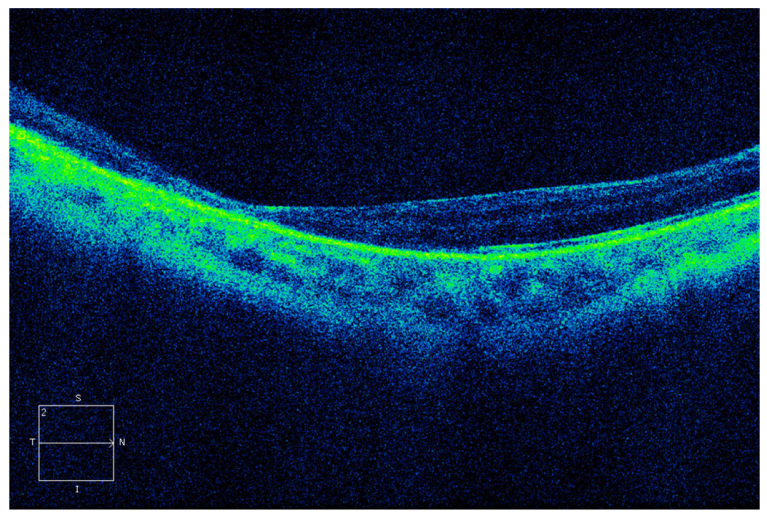

Figure 5 Optical coherence tomography image of the right eye 8 months after injury. and graft rejection could be high because of multiple irregular wounds elongating to the sclera in his eye at the time of vitrectomy. The patient was therefore referred to our clinic for retinal intervention prior to keratoplasty.

We performed a $23 \mathrm{G}$ vitrectomy because the transconjunctival system would be less invasive for the damaged conjunctiva and the two-step entry maneuvers would be less invasive and favorable for the eye with multiple penetrating wounds. We performed vitrectomy assisted by combined endoscopy and a wide-angle viewing system to overcome the poor corneal conditions, which made it impossible to observe the inside of the eye with the floating contact lens on the cornea.

The wide-angle viewing system consisted of an indirect ophthalmoscopic system and an image inverter system. One of the advantages of the wide-angle viewing system is that it allowed us to observe a wide field of the fundus in spite of a small pupil because of its indirect ophthalmoscopic nature. However, the image under the system was small. Furthermore, to observe or manipulate the periphery, indentation of the sclera was inevitable. In this case, the posterior part of the eye could be observed through the localized less damaged corneal portion, and core vitrectomy could be performed in association with the wide-angle viewing system. Furthermore, it enabled us to observe inside the eye clearly under air-filled conditions.

Endoscopy allowed us to observe the peripheral part of the retina, vitreous base, pars plana, and pars plicata, without manipulation of the anterior chamber, as well as scleral depression which could cause fluid leakage or hemorrhage from penetrating wounds in an open eye injury. In this case, shaving of the vitreous base and releasing incarceration of the vitreous into the scleral-penetrating wounds were conducted under endoscopic control without scleral indentation. Imaging with modern endoscopy is as clear as that with microscopy and is much larger than that with a microscope or a wide-angle viewing system, although the observation field is narrow and the view is nonstereoscopic. The enlarged clear image with an endoscope can facilitate the detection of a retinal break not identified preoperatively. ${ }^{7}$ A tiny retinal break was found and treated under endoscopic view in this case. The combination of endoscopy and a wide-angle viewing system made up for the deficits of each technique.

There are many controversies regarding the correct timing of vitreoretinal intervention in open eye globe injuries. Recent reports have advocated early intervention, which could avoid the risks of proliferative vitreoretinopathy. 
However, an early vitrectomy has risks of fluid leakage from the wound, intraoperative bleeding, and poor visualization of the vitreous cavity. Small gauge vitrectomy assisted by combined endoscopy and a wide-angle viewing system, which seems to be less invasive and well visualized, might minimize the risks of early intervention.

\section{Conclusion}

We describe a case of severe penetrating corneal injury that was successfully treated with a 23 G vitrectomy using an ophthalmic endoscope and a wide-angle viewing system prior to keratoplasty. A $23 \mathrm{G}$ vitrectomy assisted by combined endoscopy and a wide-angle viewing system could be advantageous in managing visualization constraints due to penetrating trauma.

\section{Acknowledgment}

The authors wish to thank Dr Chie Sotozono for performing the successful keratoplasty.

\section{Disclosure}

The authors report no conflicts of interest in this work.

\section{References}

1. Eckardt C. A new temporary keratoprosthesis for pars plan vitrectomy. Retina. 1987;7:34-37.

2. Koening SB, McDonald HR, Williams GA, Abrams GW. Penetrating keratoplasty after placement of a temporary ketatoprosthesis during pars plana vitrectomy. Am J Ophthalmol. 1986;102:45-49.

3. Kuhn F, Witherspoon CD, Morris RE. Endoscopic surgery vs temporary keratoprosthesis vitrectomy. Arch Ophthalmol. 1991;109:768.

4. Garcia-Valenzuela E, Blair NP, Shapiro MJ, et al. Outcome of vitreoretinal surgery and penetrating keratoplasty using temporary keratoprosthesis. Retina. 1999;19:424-429.

5. Ben-nun J. Cornea sparing by endoscopically guided vitreoretinal surgery. Ophthalmology. 2001;108:1465-1470.

6. Kawashima M, Kawashima S, Dogru M, Inoue M, Shimazaki J. Endoscopy-guided vitreoretinal surgery following penetrating corneal injury: a case report. Clin Ophthalmol. 2010;4:895-898.

7. Kita M, Yoshimura N. Endoscope-assisted vitrectomy in the management of pseudophakic and aphakic retinal detachments with undetected retinal breaks. Retina. 2011;31:1347-1351.
Clinical Ophthalmology

\section{Publish your work in this journal}

Clinical Ophthalmology is an international, peer-reviewed journal covering all subspecialties within ophthalmology. Key topics include: Optometry; Visual science; Pharmacology and drug therapy in eye diseases; Basic Sciences; Primary and Secondary eye care; Patient Safety and Quality of Care Improvements. This journal is indexed on

Submit your manuscript here: http://www.dovepress.com/clinical-ophthalmology-journal

\section{Dovepress}

PubMed Central and CAS, and is the official journal of The Society of Clinical Ophthalmology (SCO). The manuscript management system is completely online and includes a very quick and fair peer-review system, which is all easy to use. Visit http://www.dovepress.com/ testimonials.php to read real quotes from published authors. 